OPEN ACCESS

Edited by:

Cheng Yang,

Fudan University, China

Reviewed by:

Guillaume Hoeffel,

U1104 Centre d'immunologie de Marseille-Luminy (CIML) (INSERM),

France

Yinghong $\mathrm{Hu}$,

Emory University, United States

*Correspondence:

Bi-Cheng Liu

liubc64@163.com

${ }^{t}$ These authors have contributed equally to this work

Specialty section:

This article was submitted to Molecular Innate Immunity, a section of the journal

Frontiers in Immunology

Received: 17 March 2021 Accepted: 07 May 2021

Published: 20 May 2021

Citation:

Wen $Y$, Yan $H-R$, Wang $B$ and

Liu B-C (2021) Macrophage

Heterogeneity in Kidney

Injury and Fibrosis.

Front. Immunol. 12:681748. doi: 10.3389/fimmu.2021.681748

\section{Macrophage Heterogeneity in Kidney Injury and Fibrosis}

\author{
Yi Wen ${ }^{\dagger}$, Hong-Ru Yan ${ }^{\dagger}$, Bin Wang and Bi-Cheng Liu * \\ Department of Nephrology, Zhongda Hospital, Southeast University School of Medicine, Nanjing, China
}

Kidney macrophages are central in kidney disease pathogenesis and have therapeutic potential in preventing tissue injury and fibrosis. Recent studies highlighted that kidney macrophages are notably heterogeneous immune cells that fulfill opposing functions such as clearing deposited pathogens, maintaining immune tolerance, initiating and regulating inflammatory responses, promoting kidney fibrosis, and degrading the extracellular matrix. Macrophage origins can partially explain macrophage heterogeneity in the kidneys. Circulating Ly6C $C^{+}$monocytes are recruited to inflammatory sites by chemokines, while self-renewed kidney resident macrophages contribute to kidney repair and fibrosis. The proliferation of resident macrophages or infiltrating monocytes provides an alternative explanation of macrophage accumulation after kidney injury. In addition, dynamic Ly6C expression on infiltrating monocytes accompanies functional changes in handling kidney inflammation and fibrosis. Mechanisms underlying kidney macrophage heterogeneity, either by recruiting monocyte subpopulations, regulating macrophage polarization, or impacting distinctive macrophage functions, may help develop macrophage-targeted therapies for kidney diseases.

Keywords: macrophage heterogeneity, resident macrophage, kidney, inflammation, fibrosis

\section{INTRODUCTION}

Macrophage plays an important role in kidney disease pathogenesis and is a potential therapeutic target for kidney injury and fibrosis. Kidney macrophage subpopulations can either promote or prevent the extracellular matrix deposition in the kidney, drawing the possibility of reversing kidney fibrosis (1). However, the functionally opposing macrophage subpopulations rising ambivalence in understanding macrophage activities during kidney injury and fibrosis, disturbing the development of macrophage-targeted therapies (2). Studies have focused on macrophage functional diversities mechanisms and applied novel approaches in precisely identifying macrophage subpopulations. Therefore, intriguing questions have arisen, such as the macrophage origin (kidney-resident macrophages vs. circulating monocyte precursors), macrophage differentiation (oversimplified M1/M2 categorization vs. newly subsets defined by cell surface markers and single-cell RNAsequence), and their effector functions in the pathogenesis of kidney diseases. 


\section{MACROPHAGE HETEROGENEITY}

Macrophage heterogeneity attracts attention since the discovery of macrophages. Following studies expand macrophage heterogeneity definition depending on the origin, cell surface markers, and cytokines secretion (3). Macrophages obtain distinct phenotypes under physiological conditions and differentiate into functional phenotypes in response to pathological stimulation. According to their cooperation with distinct $\mathrm{T}$ cell subsets, macrophages have generally been classified either into classical M1 or alternative M2 macrophages. M1 macrophages are characterized by proinflammatory effects and engage with T helper 1 (Th1) cells, whereas M2 macrophages exhibit immunoregulatory efforts and intimately cooperate with $\mathrm{T}$ helper 2 (Th2) cells (4). M1 macrophage differentiation is initiated by pathogen-associated molecular patterns (PAMPs), danger-associated molecular patterns (DAMPs), and pro-inflammatory cytokines, especially under acute deleterious conditions (5). Representative functions of M1 macrophages are host defense and secretion of proinflammatory cytokines, such as tumor necrosis factor- $\alpha$ (TNF- $\alpha$ ), interleukine-1 $\beta$ (IL-1 $\beta$ ), interleukine-6 (IL-6), and interleukine-12 (IL-12). M2 macrophage is typically polarized by interleukine-4 (IL-4) and interleukine-13 (IL-13), suppressing inflammation and promoting wound repair. Recent studies further classify M2 macrophages into different subsets depending on their differentiation stimuli, markers, and functions (6). While classic M2 macrophages are classified into M2a macrophages, M2b macrophages are induced by immune complexes, toll-like receptors and/or interleukine-1 receptors (IL-1R), contributing to immunoregulation and Th2 cells activation. M2c macrophages are induced by interleukine-10 (IL-10) and glucocorticoids, participating in immunosuppression, tissue repair, and matrix deposition. The simplified paradigm of M1/M2 macrophages facilitates the studies of macrophage phenotypes and functions. However, typical M1/M2 macrophages are induced in carefully regulated circumstances in vitro and not uniformly observed under the dynamic and complex environment in vivo. In fact, macrophages appear to express M1/M2 markers simultaneously during kidney injury, and their origins largely determine the functions of renal macrophages. Lineage tracing studies demonstrated that postnatal kidney macrophages predominately originate from the yolk sac EMP and hematopoietic stem cells, and bone marrow-derived monocytes infiltrate the kidney under inflammatory conditions $(7,8)$.

\section{RENAL MACROPHAGE DISTRIBUTION DURING DEVELOPMENT, HEALTH, AND DISEASE}

Macrophages contribute to morphogenesis during organ development. In cultured kidney explants, colony-stimulating factor-1 (CSF-1) application stimulates ureteric bud branching and nephron formation (9). By binding to the membrane receptor CSF1R, CSF-1 accelerates macrophage proliferation and differentiation $(10,11)$. Macrophages infiltrate the nephrogenic zone and facilitate nephron progenitor proliferation after the transient loss of nephron progenitor cells (12). Munro et al. (13) demonstrated that macrophages directly interacted with endothelium in developing cortical nephrogenic caliber vessels. These $\mathrm{F} 4 / 80^{+} \mathrm{CD} 206^{+}$macrophages are perivascular and enriched for mRNAs associated with organogenesis. Moreover, the subpopulation of Gal3 ${ }^{\text {high }}$ myeloid cells are professional phagocytes and intermingle with pro-development $\mathrm{F} 4 / 80^{+} \mathrm{CD} 206^{+}$macrophages in the developing mouse kidney. Therefore, fetal kidney macrophages possibly facilitate organogenesis by interacting with newly forming nephrogenic blood vessels. In addition, macrophages distribute around renal tubules during kidney development (9), but the underlying mechanisms are unclear. Experimental models of invertebrate species, such as Drosophila melanogaster ( $D$. melanogaster), provide novel insights. In D. melanogaster, the Malpighian tubules are analogous to kidneys, while hemocytes are similar to macrophages. Hemocyte deposition around the developing Malpighian tubules is mediated by type IV collagen, necessary for the normal organogenesis of anterior Malpighian tubules (14). In mammalian kidneys, the renal tubular basement membrane is abundant in collagen IV and attracts macrophage recruitment $(15,16)$. However, it is difficult to extend these findings to mammalian kidneys due to the unique nephron structure.

In normal human kidneys, Marshall et al. (17) observed monocyte/macrophage distribution by immunoperoxidase staining of $\alpha$-1-antitrypsin, muramidase, and serum 22. Most positive staining cells were scattered in glomerular capillaries and intertubular blood vessels. Macrophages rarely infiltrated into tubulointerstitium except in scarring tissues. However, the generality of these findings is limited by non-specific markers. Recently, Cao et al. (18) demonstrated that $\mathrm{F} 4 / 80^{+} \mathrm{CD} 11 \mathrm{c}^{-}$ macrophages distribute throughout the renal cortex and medulla in healthy kidneys. In contrast, $\mathrm{F} 4 / 80^{+} \mathrm{CD} 11 \mathrm{c}^{+}$ mononuclear phagocytes are distributed in the renal cortex rather than the medulla under normal and injured conditions. These double-positive mononuclear phagocytes performed M1like macrophage phenotype and aggravated kidney injury during Adriamycin nephropathy. However, a large percentage of $\mathrm{CD} 11 \mathrm{c}^{+}$dendritic cells co-express $\mathrm{F} 4 / 80$ marker in healthy kidneys, the preference to determine the nature of $\mathrm{F} 4$ / $80^{+} \mathrm{CD} 11 \mathrm{c}^{+}$cells should be circumspect.

Due to a double set of arterioles and capillaries, the kidney owns a unique vascular supply and receives about $25 \%$ of the cardiac output. The renal cortex exposed continuously to large amounts of blood-derived antigens and antibodies, resulting in a high sensitivity to renal glomerular diseases (19). Glomerular macrophage accumulation is an important feature in human glomerulonephritis. Macrophage clearance decreased glomerular damage in experimental glomerulonephritis (20). Further studies targeting monocyte chemotactic molecule-1 (MCP-1) or leukocyte adhesion molecules (ICAM) successfully attenuated macrophage accumulation and kidney injury in experimental 
models $(21,22)$. Interestingly, the numbers of tubulointerstitial macrophages rather than glomerular macrophages predict renal dysfunction $(23,24)$. Our previous studies found that tubular epithelial exosomes contribute to macrophage infiltration and activation, providing a novel insight into tubulointerstitial macrophages (25-27). This review focus on macrophage heterogeneity in the kidney and excellent works have been done to card macrophage function and distribution during acute kidney injury (AKI) and chronic kidney disease (CKD) $(1,28)$.

\section{KIDNEY RESIDENT MACROPHAGE ORIGIN AND SPECIFICITY}

Kidney-resident macrophages are in situ self-renewed and characterized by their phagocytic activities, expression of pattern recognition receptors (PRRs), and immunological regulation capacity, thus, maintaining kidney homeostasis (29). Kidney-resident macrophages derive from 3 sources: (1) yolk sac erythro-myeloid progenitors (EMP)-derived macrophages, (2) fetal liver EMP-derived macrophages, and (3) hematopoietic stem cells (HSC)-derived macrophages. The relative proportion of each progenitor dramatically changes during the development, adulthood, and damaged kidney state.

At embryonic day 12.5, kidney macrophages are $\mathrm{CD} 45^{+}$ $\mathrm{CD}_{11} \mathrm{~b}^{\text {lo }} \mathrm{F} 4 / 80^{\text {hi }} \mathrm{Ly}^{-} \mathrm{C}^{-}$cells deriving from yolk sac EMP; in contrast, $\mathrm{CD} 45^{+} \mathrm{CD}_{11 \mathrm{~b}} \mathrm{~b}^{\mathrm{hi}} \mathrm{F} / 80^{\text {lo }} \mathrm{Ly}_{6 \mathrm{C}^{+}}$monocytes are undetectable at this stage (30). Using tamoxifen-inducible Runx $1^{\text {Cre/EYFP }}$ and Csf1r ${ }^{\text {Cre/EYFP }}$ mice, fate tracing studies demonstrated that the relative proportion of yolk sac EMPderived macrophages in the kidney decrease dramatically after embryonic day 13.5 (30). Consistently, Csf1r-Cre positive yolk sac-derived macrophages represent a minimal proportion of kidney macrophages after postnatal, possibly due to their dilution by the later arrival of fetal liver EMP-derived and HSC-derived macrophages (7). Sheng et al. (8) provided evidence of HSC-derived kidney macrophages using tamoxifen-inducible $c$-Kit ${ }^{\text {Cre/EYFP }}$ mice. They further concluded that HSC precursors rather than EMPs are the source of kidney resident macrophages (8). However, the non-specific expression of $c$-Kit-Cre makes this conclusion debatable $(31,32)$. As HSCs transiently expressing Flt3-cre (33), Epelman et al. (7) distinguished the origin of HSCderived monocytes and EMP-derived monocytes using Flt $3^{\mathrm{Cre} / \mathrm{GFP}}$ mice and found their equal contribution to the pool of resident macrophages. In contrast, Hoeffel et al. (30) demonstrated that fetal liver EMP-derived $\mathrm{c}-\mathrm{Myb}^{+}$monocytes are the predominant source of kidney resident macrophages. Thus, further studies based on fate-mapping studies must concern the limitations of genetic models, and single-cell RNA-sequence classifies ability worth more attention. While the kidney exposes to circulating monocytes throughout the development and adulthood, the kidney resident macrophages are mainly EMP- and HSC-derived rather than bone marrowderived $(7,8,30,32,34)$, partially explained by the niche competition hypothesis (35). Recent studies found that under certain types of kidney disease, expanded macrophages derive from the subset of resident macrophages, especially yolk sacderived macrophages $(36,37)$. Ide et al. (37) demonstrated that CX3CR $1^{+}$yolk sac-derived macrophages have a higher proliferating capacity and progressively expand in number in older mice kidneys. Kidney resident macrophage proliferation contributes to the proangiogenic and pro-inflammatory environment after ischemic AKI and is confirmed by staining with $\mathrm{Ki67}$ or $\operatorname{BrdU}(36,38)$.

Kidney resident macrophages monitor trans-endothelial transport of circulating immune complexes and regulate the infiltration of lymphocytes and neutrophils (39). Using an unbiased flow cytometry approach, Kawakami et al. (40) classified kidney resident mononuclear phagocytes into five distinct subpopulations according to their cell surface markers, including $\mathrm{CD}_{11} \mathrm{~b}^{\mathrm{hi}} \mathrm{CD} 11 \mathrm{c}^{\mathrm{hi}}, \mathrm{CD} 11 \mathrm{~b}^{\text {hi }} \mathrm{CD} 11 \mathrm{c}^{\mathrm{lo}}, \mathrm{CD} 11 \mathrm{~b}^{\text {int }}$ CD $11 c^{\text {int }}, \mathrm{CD}_{11} \mathrm{~b}^{\text {lo }} \mathrm{CD} 11 \mathrm{c}^{\text {hi }}$, and $\mathrm{CD} 11 \mathrm{~b}^{-} \mathrm{CD} 11 c^{\text {int }}$. CD $11 b^{\text {int }}$ CD11 $c^{\text {int }} \mathrm{F} 4 / 80^{\text {high }}$ monocytes perform anti-inflammation effects as endogenous defenders. Kidney resident macrophages are in situ self-renewal and minimally differentiated from circulating monocytes after ischemic AKI. However, bone marrow-derived monocytes can replenish the kidney resident macrophages when they are depleted using polyinosinic/ polycytidylic acid (poly I:C), consistent with the niche competition hypothesis (38). Interestingly, kidney resident macrophages lack major histocompatibility complex class II (MHCII) expression in the repair phase after AKI, a phenotype occurring during the nephrogenesis, and enrich Wnt ligands production, such as Axin2, Tcf4, and Jun (38). In ischemic AKI, C-C chemokine receptor type 2 (CCR2) deficiency alleviates circulating $\mathrm{Ly} 6 \mathrm{C}^{+}$macrophage recruitment and kidney injury and augments interstitial accumulation of $\mathrm{Ly} 6 \mathrm{C}^{-}$embryonic yolk sac-derived resident macrophages and kidney fibrosis in late phases (41). Clodronate Liposome-induced macrophage depletion attenuates kidney injury and fibrosis, which can be restored by adoptive transfer of Ly6C $\mathrm{C}^{-}$macrophages from injured wide type kidneys. While Ly6C $\mathrm{C}^{-}$macrophage-derived cytokines facilitate the fibroblast-myofibroblast differentiation in vivo and in vitro, direct evidence targeting the trans-differentiation from $\mathrm{Ly}^{6} \mathrm{C}^{-}$ macrophages to myofibroblasts remains missing (41). Similarly, CX3CR $1^{+}$resident renal phagocytes amplify leukocyte infiltration in an NLRP3-dependent manner in contrastinduced acute kidney injury (42). Accumulation of infiltrating and resident macrophages augments in autosomal dominant polycystic kidneys. In unilateral nephrectomy accelerated $P k d 1$ mice, lrf5 expression in resident macrophages aggravates cystic disease severity by producing pro-inflammatory cytokines (43). In ischemia-reperfusion injury (IRI) accelerated cystic mice, the phenotype of kidney resident macrophages transfers from $\mathrm{F} 4 / 80^{\text {high }} \mathrm{CD} 11 \mathrm{C}^{\text {low }}$ to $\mathrm{F} 4 / 80^{\text {high }} \mathrm{CD} 11 \mathrm{C}^{\text {high }}$, and reappearance of juvenile-like resident macrophages correlated with the accelerated cyst formation (44).

However, kidney resident macrophages also perform protective effects during acute and chronic kidney disease. 
Park et al. (45) found renal repair after ischemic AKI in mice lacking kidney resident $\mathrm{CD}_{4} 5^{+} \mathrm{Ly}_{6 \mathrm{G}}^{-} \mathrm{F} 4 / 80^{\text {high }} \mathrm{CD} 11 \mathrm{~b}^{\text {int }}$ macrophages but containing infiltrating $\mathrm{CD} 45^{+} \mathrm{Ly}_{6 \mathrm{G}}^{-} \mathrm{F} 4 / 80^{\mathrm{int}}$ $\mathrm{CD} 11 \mathrm{~b}^{\text {high }}$ macrophages is delayed compared to the wide type mice. V-domain Ig suppressor of T cell activation (VISTA), an inhibitory immune checkpoint molecule, is mainly expressed by $\mathrm{CD} 45^{+} \mathrm{Ly}_{6 \mathrm{G}^{-}} \mathrm{F} 4 / 80^{\text {high }} \mathrm{CD} 11 \mathrm{~b}^{\text {int }}$ kidney resident macrophages and has the biomarker potential in distinguishing the renal macrophages (45). CX3CR1 mediated phagocytes by kidney resident macrophages initiate within the first hours during the innate host defense against Candidiasis, confirmed by CX3CR1M280 associated susceptibility to systemic candidiasis in humans (46). CD $11 b^{\text {int }} \mathrm{F} 4 / 80^{\text {bright }}$ kidney resident macrophages protect renal artery stenosis-induced kidney injury by promoting the proangiogenic environments (36). Thus, kidney resident macrophages perform diverse effects depending on the phase and injury types. Based on a better understanding of cell surface markers and Cre specificity, further strategies should be explored to maintain protective resident macrophage phenotype during kidney disease. To advance these studies toward clinic interventions in patients, we must overcome two shortages of kidney resident macrophages. Firstly, minimal information is known about the anatomy and functions of resident macrophages in human kidney. Secondly, the similarities and differences of resident macrophages between rodent models and human kidneys remain unclear.

\section{MONOCYTE AS PRECURSORS OF KIDNEY MACROPHAGES}

While circulating monocytes minimally contribute to the renal macrophage pool under homeostasis, toxic or infectious damages result in augmented recruitment of monocyte-derived macrophages into the kidney. When renal macrophage niches are ablated, peripheral monocytes rapidly differentiate and replenish kidney macrophages (Munro et al. Nature Communications 11(1):2280 DOI:10.1038/s41467-020-16158-z). Bone marrow-derived monocyte precursors can reconstitute ischemic kidney macrophages in niches when kidney resident macrophages are depleted (38). Colony-stimulating factor-1 (CSF-1) stimulates macrophage proliferation at various time phases and tissues (47). Genetic deficiency or pharmacological blockade targeting CSF-1 inhibits macrophage proliferation, therefore prolonging the tissue repair phase after AKI (48). Rodent models revealed that Ly6 $\mathrm{C}^{\text {high }}$ pro-inflammatory monocytes infiltrate early in damaged kidneys (49, 50), depending on chemokines such as CCL2, CCL5, and CX3CR1 (26, 51-53). Initial infiltrating bone marrow-derived macrophages are characterized as Ly6C high iNOS $^{+}$cells by flow cytometry in rodent kidneys, whereas late Ly6C $\mathrm{C}^{\text {low }}$ macrophages perform profibrotic M2-like effects $(49,50)$. CD11b diphtheria toxin receptor (DTR)-mediated depletion of monocyte/macrophages (50) or pharmacological blockade targeting chemokine pathways (54) attenuates kidney fibrosis, suggesting a profibrotic role of infiltrating macrophages in renal fibrogenesis. In severe IRI-induced AKI-CKD models, adoptive transplantation of $\mathrm{F} 4 / 80^{\text {int }} \mathrm{B} 7-\mathrm{H} 4^{\text {high }}$ (M2c) macrophages rather than $\mathrm{F} 4 / 80^{\text {high }} \mathrm{B} 7-\mathrm{H} 4$ int $^{\text {in } 1 \text { ) }}$ macrophages restore renal interstitial fibrosis in liposome clodronate-induced macrophage abrogated mice (55). Similarly, adoptive transfer of $\mathrm{F} 4 / 80^{+} \mathrm{CD}^{+} 01^{+}$(M2) macrophages rescue renal fibrosis in obstructed kidneys after macrophage depletion (56). Monocyte-derived kidney macrophages aggravate fibroblast activation and renal fibrosis by secreting cytokines $(1,57)$. Despite the direct and indirect profibrotic effects, bone marrow-derived macrophages can transdifferentiate into collagen-producing myofibroblasts via macrophage-myofibroblast transition (MMT) (58). Using Lyz2-Cre/Rosa26-Tomato mice, lineage tracing studies demonstrated that approximal $50 \%$ of the $\alpha \mathrm{SMA}^{+}$Collagen $^{+}$ myofibroblasts derive from $\mathrm{F} 4 / 80^{+}$Tomato $^{+}$myeloid cells (59, $60)$. In contrast, a 2018 study challenges the MMT hypothesis as bone marrow-derived myofibroblasts make a limited contribution to the myofibroblasts in the obstructed kidney (61). The conflicting results come from the identification of myeloid cells by $\mathrm{CD} 45^{+}$and myofibroblasts by $\mathrm{PDGFR}^{+}$, as unspecific markers amplify the miscalculation. Another limitation is the deficiency of markers to distinguish bone marrow-derived fibroblasts from macrophagederived myofibroblasts.

Moreover, macrophages perform diversified roles in renal fibrogenesis via secreting matrix metalloproteinases. Matrix metalloproteinases, especially macrophage-derived matrix metalloproteinase-9 (MMP-9), promote kidney fibrosis through stimulating extracellular matrix deposition $(62,63)$. In contrast, Twist 1 in infiltrating macrophages promotes extracellular matrix degradation by stimulating $\mathrm{CD}_{11 \mathrm{~b}^{+}}$ Ly6 $\mathrm{C}^{\text {low }}$-derived matrix metalloproteinase-13 (MMP-13) production (64). As $\mathrm{CD}_{11 \mathrm{~b}^{+}}$Ly6 $\mathrm{C}^{\text {high }}$ monocytes freshly infiltrate after kidney injury and represent the onset of renal inflammation, CD $11 \mathrm{~b}^{+} \mathrm{Ly}_{6} \mathrm{C}^{\text {int }}$ and $\mathrm{CD}_{1} 1 \mathrm{~b}^{+} \mathrm{Ly}_{6} \mathrm{C}^{\text {low }}$ populations expand in the phases of repair and fibrosis (41). The evidence that terminally differentiated macrophages rather than freshly infiltrating monocyte progenitors are the major players in kidney fibrogenesis suggests that kidney macrophages function varies due to disease types and time phases.

The Ly6C ${ }^{\text {low }}$ subpopulation of circulating monocytes is characterized by monitoring and phagocytosing circulating immune complexes (65). These Ly6C $\mathrm{C}^{\text {low }}$ monocytes present antigens and activate effector $\mathrm{CD} 4{ }^{+} \mathrm{T}$ lymphocytes in the glomerular capillaries (66). Circulating myeloid-derived suppressor cells (MDSCs) are immune suppressive populations and initially investigated in cancer (67). MDSCs were firstly reported to maintain cardiac transplant tolerance in rodent models (46), whereas renal MDSCs accumulation positively correlates with graft survival and kidney transplant recipients $(68,69)$. The effects of MDSCs further expand to immuneregulation and fibrogenesis during septic and metabolic kidney disease $(70,71)$, suggesting the therapeutic potential of MDSCs in acute and chronic kidney disease. 


\section{HETEROGENEITY OF HUMAN KIDNEY MACROPHAGES AND CLINICAL TRANSFORMATION}

Human monocyte/macrophages are classified into three distinct populations, including classical $\mathrm{CD}_{1} 4^{++} \mathrm{CD}_{16}{ }^{-}$subset, nonclassical $\mathrm{CD}_{14}{ }^{+} \mathrm{CD}_{1} 6^{+}$subset, and intermediate $\mathrm{CD} 14^{++}$ $\mathrm{CD} 6^{+}$subset (72). In a 35 months cohort with 94 dialysis patients, the number of classical $\mathrm{CD} 14^{++} \mathrm{CD} 16^{-}$monocytes can independently predict cardiovascular events and death (73). Rogacev et al. (74) demonstrated $\mathrm{CD} 14^{++} \mathrm{CD}^{-} 6^{-}$monocytes numbers can also predict cardiovascular events in CKD patients. Non-classical CD $14^{+} \mathrm{CD}_{16}{ }^{+}$monocytes from CKD patients express high levels of chemokines, facilitating their adhesion to vascular walls (75). Pro-inflammatory $\mathrm{CD} 14^{+} \mathrm{CD}_{16}{ }^{+}$monocytes correlate with blood vessel stiffness in predialysis CKD patients, suggesting that non-classical $\mathrm{CD}_{1} 4^{+} \mathrm{CD}_{1}{ }^{+}$subset damage endothelial cells (76). However, most clinical studies lack direct evidence of macrophage populations inside the kidney, and the predictive ability of human macrophage populations in kidney injury and fibrosis requires further investigations.

To expand experimental knowledge of macrophages to clinical applications, we still have to overcome several obstacles. Firstly, available data of human kidney macrophages is significantly insufficient, especially the dynamic changes of macrophage function and subpopulations. Secondly, CD $14^{++} \mathrm{CD} 16^{-}$and $\mathrm{CD} 14^{++} \mathrm{CD} 16^{+}$monocytes resemble mouse $\mathrm{Ly} 6 \mathrm{C}^{+}$inflammatory monocytes, whereas $\mathrm{CD} 14^{+} \mathrm{CD} 16^{+}$monocytes share phenotypic features with $\mathrm{Ly}^{-} \mathrm{C}^{-}$anti-inflammatory monocytes and adhering vascular endothelium (77). As classical CD14 ${ }^{++} \mathrm{CD}^{-} 6^{-}$subset is known for the phagocytic capability, the overlapping and sometimes conflicting features of human and murine monocytes require further investigation. Thirdly, the functionality of kidney macrophages is dynamically variable and affected by the nature of kidney diseases. Thus, clinic translation must address the characteristics of kidney macrophages in different types and phases of the disease (Table 1).

Nevertheless, characterized macrophage recruitment and maturation pathways are also appliable in patients with kidney diseases. Renoprotective effects of interventions targeting CCL2/ CCR2 have been confirmed in rodent models of kidney inflammation and fibrosis $(26,41)$. Consistently, CCR2 inhibitor CCX140-B further attenuates albuminuria levels in patients with type 2 diabetes in addition to the standard renin-angiotensin system (RAS) blocking therapies (84). Although the definite effects of CSF1R in macrophage recruitment and proliferation, the role of CSF1R inhibitors and neutralizing antibodies have not been tested in clinical trials to treat kidney diseases. The JAK-STAT pathway regulates macrophage activation and renal function decline in patients with type 2 diabetic nephropathy (85). JAK1 and JAK2 inhibition by Bariticinib decreases albuminuria levels in patients with diabetic kidney diseases (DKD) (86). Our recent studies found that macrophage-derived extracellular vesicles are kidney-targeted drug carriers and worth further expansion into clinical trials (87).

\section{SINGLE-CELL RNA-SEQUENCING REVOLUTION}

After the qualitative leap from the oversimplified M1/M2 paradigm to individual subpopulations identified by cell surface markers, single-cell RNA-sequencing (scRNA-seq) categorizes macrophages by their function and phenotype, revealing the continuum and complexity of macrophages during the development and pathogenesis of kidney disease $(88,89)$. Zimmerman et al. (80) identified $C 1 q$ expression as a novel marker of resident macrophage clusters in mouse kidneys, and $C 1 q$ expressing clusters in other species were verified by the expression of $C d 74, C d 81$, and Apoe. In a rodent model of rhabdomyolysis-induced AKI, F4/80 ${ }^{\text {low }} \mathrm{CD} 11 \mathrm{~b}^{\text {high }}$ Ly $6 b^{\text {high }}$ CD $206^{\text {low }}$ pro-inflammatory macrophages infiltrate early

TABLE 1 | Macrophage markers in human and mouse.

\begin{tabular}{|c|c|c|c|c|}
\hline Markers & Gene & Species & Protein Type & Reference \\
\hline $\mathrm{B} 7-\mathrm{H} 4$ & VTCN1 & Human, Mouse & Cell Membrane Receptor & (55) \\
\hline B7-H5 (VISTA) & VSIR & Human, Mouse & Cell Membrane Cytokine & (45) \\
\hline CD11b & ITGAM & Human, Mouse & Cell Membrane Receptor & $(30,36,41,45,64)$ \\
\hline CDIlc & ITGAX & Human, Mouse & Cell Membrane Receptor & $(18,44,78)$ \\
\hline CDI4 & CD 14 & Human, Mouse & Cell Membrane Receptor & $(72-76)$ \\
\hline CD16 & FCGR3A & Human, Mouse & Cell Membrane Receptor & $(72-77)$ \\
\hline CD45 & PTPRC & Human, Mouse & Cell Membrane Receptor & $(30,45)$ \\
\hline CD115 & CSF1R & Human, Mouse & Cell Membrane Receptor & (30) \\
\hline CD192 & CCR2 & Human, Mouse & Cell Membrane Receptor & (79) \\
\hline CD206 & MRC1 & Human, Mouse & Cell Membrane Receptor & $(13,80)$ \\
\hline CCRL1 & CX3CR1 & Human, Mouse & Cell Membrane Receptor & $(37,42)$ \\
\hline $\mathrm{F} 4 / 80$ & ADGRE1 & Human, Mouse & Cell Membrane Receptor & $(13,18,30,36,40,44,45,55,56,81,82)$ \\
\hline iNOS & NOS2 & Human, Mouse & Cytoplasm Enzyme & $(49,50)$ \\
\hline Ly6C & Ly6c1 & Mouse & Cell Membrane Receptor & $(30,41,49,50,77)$ \\
\hline MHC class ॥ & $\mathrm{MHCll}$ & Human Mouse & Cell Membrane Receptor & $(38)$ \\
\hline MCP-1 & CCL2 & Human Mouse & Secreted Cytokine & $(26,79)$ \\
\hline SCARD1 & CD68 & Human Mouse & Cell Membrane Receptor & (83) \\
\hline TNF- $\alpha$ & TFN & Human, Mouse & Cell Membrane Cytokine & (57) \\
\hline
\end{tabular}


after rhabdomyolysis, while F4/80 $0^{\text {high }} \mathrm{CD} 11 \mathrm{~b}^{+}$Ly6b ${ }^{\text {low }} \mathrm{CD} 206^{\text {high }}$ reparative macrophages are dominant at late phase. However, the scRNA-seq analysis demonstrated that signal pathways do not precisely match macrophage phenotypes and the existence of individual subpopulations simultaneously expressing heterogeneous markers (81). At the late phase after ischemic AKI, macrophages expressing $\mathrm{Ccl} 2$ and $\mathrm{Ccr} 2$ infiltrate around the $\mathrm{Vcam}^{+}$damaged tubules (79). By evaluating the typical C1qa, Cd74, and Adgre1 expression, macrophages are defined as the predominant immune cells in diabetic glomeruli and mainly M1like macrophages (90). The scRNA-seq analysis revealed that both extent and levels of $A x l$ expression increased in $\mathrm{F} 4 / 80^{+}$macrophages from rejecting allografts compared to tolerized kidneys; and $A x l$ promotes the differentiation of intra-graft myeloid cells towards pro-inflammatory phenotypes after transplantation (82). Moreover, the combination of scRNA-seq and lineage tracing technique attracts particular attention. Lineage tracing can elucidate the clonal relationships during development and differentiation, enable lifecycle monitoring. In contrast, scRNA-seq can identify exact cell types but unable to determine the lineage relationships. Thus, integrating scRNA-seq and lineage tracing will provide extra information about cell types, development, and differentiation in a longstanding pattern.
Stewart et al. (78) demonstrated that human kidney monocyte phagocytes (MNPs) expressing ITGAX and HLA-DRA are categorized into four distinct clusters ( $\mathrm{MNPa}$ to $\mathrm{MNPd}$ ). $\mathrm{MNPa}$ subpopulation expressing CD14 in mature kidney analogous classical monocytes, whereas MNPb expressing CD16 is transcriptionally similar to non-classical monocytes. Consistently, $\mathrm{CD}_{14}{ }^{+} \mathrm{CD} 8^{+}$monocyte/macrophages are the most abundant immune cells in urine and account for one-third of urinary clusters. These monocyte/macrophages are enriched in genes related to antigen presentation and macrophage activation and further classified into $\mathrm{CD}^{+} 6^{+}$and $\mathrm{CD}^{-} 6^{-}$subpopulations (83).

\section{CONCLUSIONS AND PERSPECTIVES}

Macrophages are recruited by chemokines and contribute to the pathogenesis of kidney injury, repair, and fibrosis. Despite the wide application of the oversimplified pro-inflammatory M1 and antiinflammatory M2 macrophage paradigm, macrophage complexity in origin, phenotype, and function has attracted attention. Macrophage subpopulations were distinguished by combining cell surface markers and using novel single-cell RNA sequence technology to explore macrophage contribution in tissue injury,

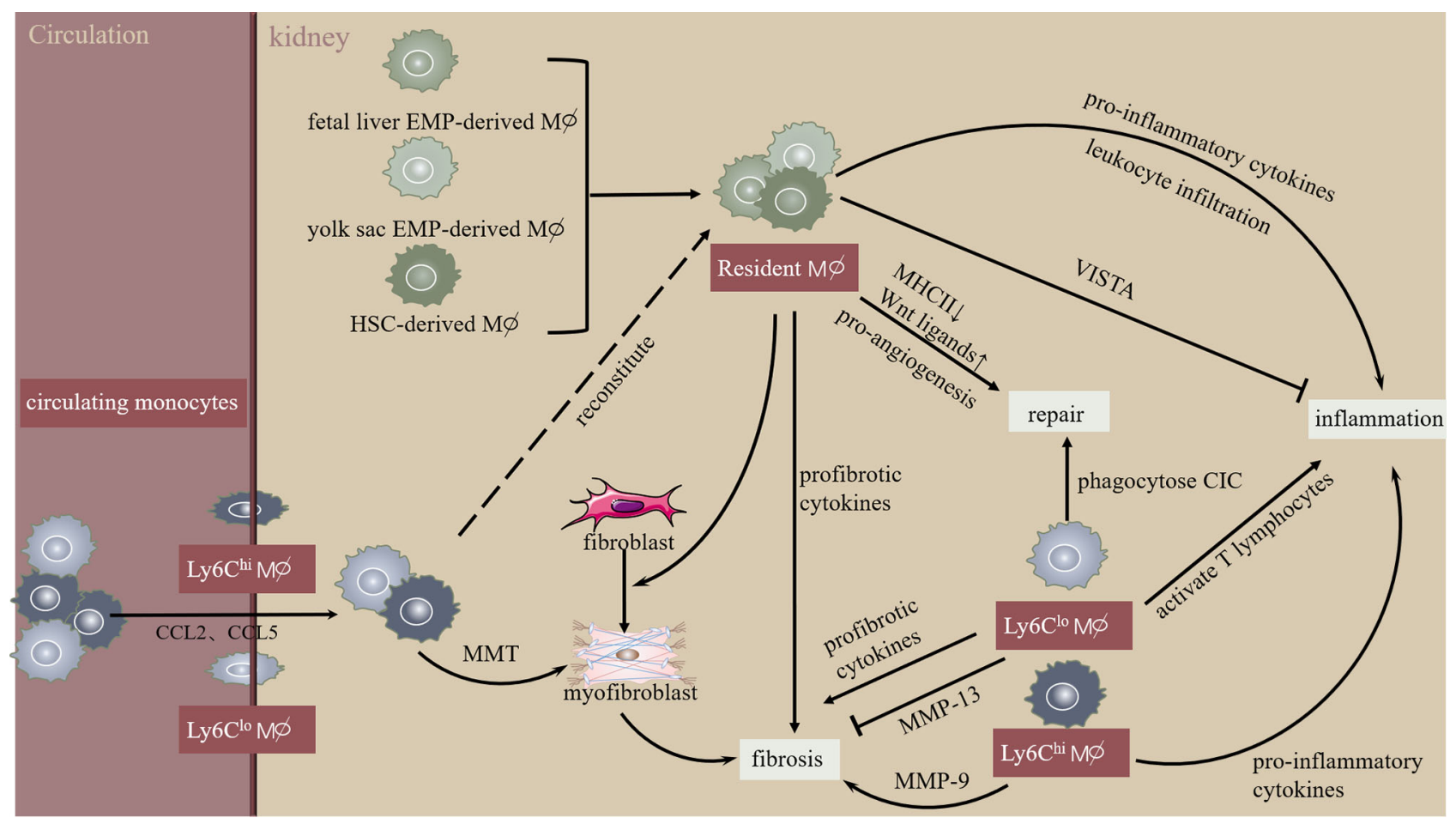

FIGURE 1 | Macrophage heterogeneity during initiation and progression of kidney injury and fibrosis. Kidney resident macrophages derive from multi-sources and monitor trans-endothelial transport of circulating immune complexes. In the initial phase of kidney injury, resident macrophages stimulate leukocyte infiltration and cytokine secretion. Interestingly, kidney resident macrophages express V-domain Ig suppressor of T cell activation (VISTA), an inhibitory immune checkpoint molecule. Increased chemokines (CCL2, CCL5) promote circulating monocyte chemotaxis into the kidney, developing into infiltrating Ly-6C ${ }^{\text {hi }}$ macrophages exhibiting pro-inflammatory phenotype or macrophage-myofibroblast transition (MMT). Similarly, infiltrating Ly6C ${ }^{\text {low }}$ macrophages promote kidney inflammation and fibrosis via activating T lymphocytes or pro-inflammatory cytokines. However, Ly6C ${ }^{\text {hi }}$ macrophages inhibit kidney fibrosis by producing MMP-13. Overall, these mechanisms lead to extracellular matrix dynamic homeostasis during the resolution of kidney injury and fibrosis. 
regeneration, and fibrosis. Our data and others have confirmed the therapeutic potential of macrophage pathways in acute and chronic kidney diseases; however, the functionally opposing macrophage subpopulations require incisive and tissue-specific strategies (Figure 1). Moreover, an in-depth understanding of the specialty and commonality in scRNA-seq defined macrophage clusters requires further investigation.

\section{AUTHOR CONTRIBUTIONS}

YW and H-RY contributed equally to the writing of the manuscript. BW contributed to the figure of the manuscript.

\section{REFERENCES}

1. Tang PM, Nikolic-Paterson DJ, Lan HY. Macrophages: Versatile Players in Renal Inflammation and Fibrosis. Nat Rev Nephrol (2019) 15(3):144-58. doi: 10.1038/s41581-019-0110-2

2. Wen Y, Crowley SD. The Varying Roles of Macrophages in Kidney Injury and Repair. Curr Opin Nephrol Hypertens (2020) 29(3):286-92. doi: 10.1097/ MNH.0000000000000595

3. Cao Q, Wang Y, Harris DC. Macrophage Heterogeneity, Phenotypes, and Roles in Renal Fibrosis. Kidney Int Suppl (2011) (2014) 4(1):16-9. doi: 10.1038/kisup.2014.4

4. Lee S, Huen S, Nishio H, Nishio S, Lee HK, Choi BS, et al. Distinct Macrophage Phenotypes Contribute to Kidney Injury and Repair. J Am Soc Nephrol (2011) 22(2):317-26. doi: 10.1681/ASN.2009060615

5. Lv LL, Tang PM, Li CJ, You YK, Li J, Huang XR, et al. The Pattern Recognition Receptor, Mincle, is Essential for Maintaining the M1 Macrophage Phenotype in Acute Renal Inflammation. Kidney Int (2017) 91 (3):587-602. doi: 10.1016/j.kint.2016.10.020

6. Chen T, Cao Q, Wang Y, Harris DCH. M2 Macrophages in Kidney Disease: Biology, Therapies, and Perspectives. Kidney Int (2019) 95(4):760-73. doi: 10.1016/j.kint.2018.10.041

7. Epelman S, Lavine KJ, Beaudin AE, Sojka DK, Carrero JA, Calderon B, et al. Embryonic and Adult-Derived Resident Cardiac Macrophages are Maintained Through Distinct Mechanisms at Steady State and During Inflammation. Immunity (2014) 40(1):91-104. doi: 10.1016/j.immuni. 2013.11.019

8. Sheng J, Ruedl C, Karjalainen K. Most Tissue-Resident Macrophages Except Microglia Are Derived From Fetal Hematopoietic Stem Cells. Immunity (2015) 43(2):382-93. doi: 10.1016/j.immuni.2015.07.016

9. Rae F, Woods K, Sasmono T, Campanale N, Taylor D, Ovchinnikov DA, et al. Characterisation and Trophic Functions of Murine Embryonic Macrophages Based Upon the Use of a Csf1r-EGFP Transgene Reporter. Dev Biol (2007) 308(1):232-46. doi: 10.1016/j.ydbio.2007.05.027

10. Dai XM, Ryan GR, Hapel AJ, Dominguez MG, Russell RG, Kapp S, et al. Targeted Disruption of the Mouse Colony-Stimulating Factor 1 Receptor Gene Results in Osteopetrosis, Mononuclear Phagocyte Deficiency, Increased Primitive Progenitor Cell Frequencies, and Reproductive Defects. Blood (2002) 99(1):111-20. doi: 10.1182/blood.v99.1.111

11. Mouchemore KA, Pixley FJ. CSF-1 Signaling in Macrophages: Pleiotrophy Through Phosphotyrosine-Based Signaling Pathways. Crit Rev Clin Lab Sci (2012) 49(2):49-61. doi: 10.3109/10408363.2012.666845

12. Muthukrishnan SD, Ryzhov S, Karolak M, Mukherjee E, Sims-Lucas S, Oxburgh L. A Macrophage-Based Regenerative Response to Fetal Kidney Damage. Mech Dev (2017) 145:S50-0. doi: 10.1016/j.mod. 2017.04.094

13. Munro DAD, Wineberg Y, Tarnick J, Vink CS, Li Z, Pridans C, et al. Macrophages Restrict the Nephrogenic Field and Promote Endothelial Connections During Kidney Development. Elife (2019) 8:e43271. doi: $10.7554 /$ eLife.43271
B-CL conceived the concept and contributed to the writing of the manuscript. All authors contributed to the article and approved the submitted version.

\section{FUNDING}

This work was supported by China National Natural Science Foundation (8203000544, 81720108007) and the key research project of the Ministry of China Science and Technology (2018YFC1314000) to B-CL as PI, and National Natural Science Foundation of China (81900623), and the Natural Science Foundation of Jiangsu Province (BK20190349) to YW as PI.

14. Bunt S, Hooley C, Hu N, Scahill C, Weavers H, Skaer H. Hemocyte-Secreted Type IV Collagen Enhances BMP Signaling to Guide Renal Tubule Morphogenesis in Drosophila. Dev Cell (2010) 19(2):296-306. doi: 10.1016/ j.devcel.2010.07.019

15. Schnoor M, Cullen P, Lorkowski J, Stolle K, Robenek H, Troyer D, et al. Production of Type VI Collagen by Human Macrophages: A New Dimension in Macrophage Functional Heterogeneity. J Immunol (2008) 180(8):5707-19. doi: 10.4049/jimmunol.180.8.5707

16. Abrahamson DR, Leardkamolkarn V. Development of Kidney Tubular Basement Membranes. Kidney Int (1991) 39(3):382-93. doi: 10.1038/ ki.1991.50

17. Marshall RJ, MacIver AG. The Monocyte/Macrophage Population of the Normal Human Kidney. J Pathol (1984) 143(4):275-80. doi: 10.1002/ path.1711430407

18. Cao Q, Wang Y, Wang XM, Lu J, Lee VW, Ye Q, et al. Renal F4/80+ CD11c+ Mononuclear Phagocytes Display Phenotypic and Functional Characteristics of Macrophages in Health and in Adriamycin Nephropathy. J Am Soc Nephrol (2015) 26(2):349-63. doi: 10.1681/ASN.2013121336

19. Couser WG. Basic and Translational Concepts of Immune-Mediated Glomerular Diseases. J Am Soc Nephrol (2012) 23(3):381-99. doi: 10.1681/ ASN.2011030304

20. Duffield JS, Tipping PG, Kipari T, Cailhier JF, Clay S, Lang R, et al. Conditional Ablation of Macrophages Halts Progression of Crescentic Glomerulonephritis. Am J Pathol (2005) 167(5):1207-19. doi: 10.1016/ S0002-9440(10)61209-6

21. Tesch GH, Schwarting A, Kinoshita K, Lan HY, Rollins BJ, Kelley VR. Monocyte Chemoattractant Protein-1 Promotes Macrophage-Mediated Tubular Injury, But Not Glomerular Injury, in Nephrotoxic Serum Nephritis. J Clin Invest (1999) 103(1):73-80. doi: 10.1172/JCI4876

22. Chow FY, Nikolic-Paterson DJ, Ozols E, Atkins RC, Tesch GH. Intercellular Adhesion Molecule-1 Deficiency is Protective Against Nephropathy in Type 2 Diabetic db/db Mice. J Am Soc Nephrol (2005) 16(6):1711-22. doi: 10.1681/ ASN.2004070612

23. Silva GE, Costa RS, Ravinal RC, Ramalho LN, Reis MA, Moyses-Neto M, et al. Renal Macrophage Infiltration is Associated With a Poor Outcome in IgA Nephropathy. Clinics (Sao Paulo) (2012) 67(7):697-703. doi: 10.6061/clinics/ 2012(07)01

24. Liu D, Xu M, Ding LH, Lv LL, Liu H, Ma KL, et al. Activation of the Nlrp3 Inflammasome by Mitochondrial Reactive Oxygen Species: A Novel Mechanism of Albumin-Induced Tubulointerstitial Inflammation. Int J Biochem Cell Biol (2014) 57:7-19. doi: 10.1016/j.biocel.2014.09.018

25. Li ZL, Lv LL, Tang TT, Wang B, Feng Y, Zhou LT, et al. HIF-1alpha Inducing Exosomal microRNA-23a Expression Mediates the Cross-Talk Between Tubular Epithelial Cells and Macrophages in Tubulointerstitial Inflammation. Kidney Int (2019) 95(2):388-404. doi: 10.1016/j.kint.2018.09.013

26. Lv LL, Feng Y, Wen Y, Wu WJ, Ni HF, Li ZL, et al. Exosomal CCL2 From Tubular Epithelial Cells is Critical for Albumin-Induced Tubulointerstitial Inflammation. J Am Soc Nephrol (2018) 29(3):919-35. doi: 10.1681/ ASN.2017050523 
27. Lv LL, Feng Y, Wu M, Wang B, Li ZL, Zhong X, et al. Exosomal miRNA-19b3p of Tubular Epithelial Cells Promotes M1 Macrophage Activation in Kidney Injury. Cell Death Differ (2020) 27(1):210-26. doi: 10.1038/s41418-019-0349-y

28. Cao Q, Harris DC, Wang Y. Macrophages in Kidney Injury, Inflammation, and Fibrosis. Physiol (Bethesda) (2015) 30(3):183-94. doi: 10.1152/ physiol.00046.2014

29. Munro DAD, Hughes J. The Origins and Functions of Tissue-Resident Macrophages in Kidney Development. Front Physiol (2017) 8:837. doi: 10.3389/fphys.2017.00837

30. Hoeffel G, Chen J, Lavin Y, Low D, Almeida FF, See P, et al. C-Myb(+) Erythro-Myeloid Progenitor-Derived Fetal Monocytes Give Rise to Adult Tissue-Resident Macrophages. Immunity (2015) 42(4):665-78. doi: 10.1016/ j.immuni.2015.03.011

31. Gomez Perdiguero E, Klapproth K, Schulz C, Busch K, Azzoni E, Crozet L, et al. Tissue-Resident Macrophages Originate From Yolk-Sac-Derived Erythro-Myeloid Progenitors. Nature (2015) 518(7540):547-51. doi: 10.1038/nature13989

32. Hoeffel G, Ginhoux F. Ontogeny of Tissue-Resident Macrophages. Front Immunol (2015) 6:486. doi: 10.3389/fimmu.2015.00486

33. Boyer SW, Schroeder AV, Smith-Berdan S, Forsberg EC. All Hematopoietic Cells Develop From Hematopoietic Stem Cells Through Flk2/Flt3-positive Progenitor Cells. Cell Stem Cell (2011) 9(1):64-73. doi: 10.1016/ j.stem.2011.04.021

34. Epelman S, Lavine KJ, Randolph GJ. Origin and Functions of Tissue Macrophages. Immunity (2014) 41(1):21-35. doi: 10.1016/j.immuni. 2014.06.013

35. Guilliams M, Scott CL. Does Niche Competition Determine the Origin of Tissue-Resident Macrophages? Nat Rev Immunol (2017) 17(7):451-60. doi: $10.1038 /$ nri.2017.42

36. Puranik AS, Leaf IA, Jensen MA, Hedayat AF, Saad A, Kim KW, et al. KidneyResident Macrophages Promote a Proangiogenic Environment in the Normal and Chronically Ischemic Mouse Kidney. Sci Rep (2018) 8(1):13948. doi: 10.1038/s41598-018-31887-4

37. Ide S, Yahara Y, Kobayashi Y, Strausser SA, Ide K, Watwe A, et al. Yolk-SacDerived Macrophages Progressively Expand in the Mouse Kidney With Age. Elife (2020) 9:e51756. doi: 10.7554/eLife.51756

38. Lever JM, Hull TD, Boddu R, Pepin ME, Black LM, Adedoyin OO, et al. Resident Macrophages Reprogram Toward a Developmental State After Acute Kidney Injury. JCI Insight (2019) 4(2):e125503. doi: 10.1172/jci.insight.125503

39. Stamatiades EG, Tremblay ME, Bohm M, Crozet L, Bisht K, Kao D, et al. Immune Monitoring of Trans-endothelial Transport by Kidney-Resident Macrophages. Cell (2016) 166(4):991-1003. doi: 10.1016/j.cell.2016.06.058

40. Kawakami T, Lichtnekert J, Thompson LJ, Karna P, Bouabe H, Hohl TM, et al. Resident Renal Mononuclear Phagocytes Comprise Five Discrete Populations With Distinct Phenotypes and Functions. J Immunol (2013) 191(6):3358-72. doi: 10.4049/jimmunol.1300342

41. Yang Q, Wang $\mathrm{Y}$, Pei G, Deng X, Jiang H, Wu J, et al. Bone Marrow-Derived Ly6C(-) Macrophages Promote Ischemia-Induced Chronic Kidney Disease. Cell Death Dis (2019) 10(4):291. doi: 10.1038/s41419-019-1531-3

42. Lau A, Chung H, Komada T, Platnich JM, Sandall CF, Choudhury SR, et al. Renal Immune Surveillance and Dipeptidase-1 Contribute to ContrastInduced Acute Kidney Injury. J Clin Invest (2018) 128(7):2894-913. doi: 10.1172/JCI96640

43. Zimmerman KA, Huang J, He L, Revell DZ, Li Z, Hsu JS, et al. Interferon Regulatory Factor-5 in Resident Macrophage Promotes Polycystic Kidney Disease. Kidney360 (2020) 1(3):179-90. doi: 10.34067/KID.0001052019

44. Zimmerman KA, Song CJ, Li Z, Lever JM, Crossman DK, Rains A, et al. Tissue-Resident Macrophages Promote Renal Cystic Disease. J Am Soc Nephrol (2019) 30(10):1841-56. doi: 10.1681/ASN.2018080810

45. Park JG, Lee CR, Kim MG, Kim G, Shin HM, Jeon YH, et al. Kidney Residency of VISTA-positive Macrophages Accelerates Repair From Ischemic Injury. Kidney Int (2020) 97(5):980-94. doi: 10.1016/j.kint.2019.11.025

46. Lionakis MS, Swamydas M, Fischer BG, Plantinga TS, Johnson MD, Jaeger M, et al. CX3CR1-Dependent Renal Macrophage Survival Promotes Candida Control and Host Survival. J Clin Invest (2013) 123(12):5035-51. doi: 10.1172/ JCI71307

47. Menke J, Rabacal WA, Byrne KT, Iwata Y, Schwartz MM, Stanley ER, et al. Circulating CSF-1 Promotes Monocyte and Macrophage Phenotypes That
Enhance Lupus Nephritis. J Am Soc Nephrol (2009) 20(12):2581-92. doi: 10.1681/ASN.2009050499

48. Zhang MZ, Yao B, Yang S, Jiang L, Wang S, Fan X, et al. CSF-1 Signaling Mediates Recovery From Acute Kidney Injury. J Clin Invest (2012) 122 (12):4519-32. doi: 10.1172/JCI60363

49. Menezes S, Melandri D, Anselmi G, Perchet T, Loschko J, Dubrot J, et al. The Heterogeneity of Ly6C(hi) Monocytes Controls Their Differentiation Into iNOS(+) Macrophages or Monocyte-Derived Dendritic Cells. Immunity (2016) 45(6):1205-18. doi: 10.1016/j.immuni.2016.12.001

50. Lin SL, Castano AP, Nowlin BT, Lupher MLJr., Duffield JS. Bone Marrow Ly6Chigh Monocytes are Selectively Recruited to Injured Kidney and Differentiate Into Functionally Distinct Populations. J Immunol (2009) 183 (10):6733-43. doi: 10.4049/jimmunol.0901473

51. Rudemiller NP, Patel MB, Zhang JD, Jeffs AD, Karlovich NS, Griffiths R, et al. C-C Motif Chemokine 5 Attenuates Angiotensin II-Dependent Kidney Injury by Limiting Renal Macrophage Infiltration. Am J Pathol (2016) 186(11):284656. doi: 10.1016/j.ajpath.2016.07.015

52. Cassini MF, Kakade VR, Kurtz E, Sulkowski P, Glazer P, Torres R, et al. Mcp1 Promotes Macrophage-Dependent Cyst Expansion in Autosomal Dominant Polycystic Kidney Disease. J Am Soc Nephrol (2018) 29(10):2471-81. doi: 10.1681/ASN.2018050518

53. Chousterman BG, Boissonnas A, Poupel L, Baudesson de Chanville C, Adam J, Tabibzadeh N, et al. Ly6Chigh Monocytes Protect Against Kidney Damage During Sepsis Via a CX3CR1-Dependent Adhesion Mechanism. J Am Soc Nephrol (2016) 27(3):792-803. doi: 10.1681/ASN.2015010009

54. McRae JL, Vikstrom IB, Bongoni AK, Salvaris EJ, Fisicaro N, Ng M, et al. Blockade of the G-CSF Receptor Is Protective in a Mouse Model of Renal Ischemia-Reperfusion Injury. J Immunol (2020) 205(5):1433-40. doi: 10.4049/ jimmunol.2000390

55. Kim MG, Kim SC, Ko YS, Lee HY, Jo SK, Cho W. The Role of M2 Macrophages in the Progression of Chronic Kidney Disease Following Acute Kidney Injury. PloS One (2015) 10(12):e0143961. doi: 10.1371/ journal.pone. 0143961

56. Shen B, Liu X, Fan Y, Qiu J. Macrophages Regulate Renal Fibrosis Through Modulating TGFbeta Superfamily Signaling. Inflammation (2014) 37 (6):2076-84. doi: 10.1007/s10753-014-9941-y

57. Wen Y, Lu X, Ren J, Privratsky JR, Yang B, Rudemiller NP, et al. KLF4 in Macrophages Attenuates TNFalpha-Mediated Kidney Injury and Fibrosis. J Am Soc Nephrol (2019) 30(10):1925-38. doi: 10.1681/ASN. 2019020111

58. Phua YL, Martel N, Pennisi DJ, Little MH, Wilkinson L. Distinct Sites of Renal Fibrosis in Crim1 Mutant Mice Arise From Multiple Cellular Origins. J Pathol (2013) 229(5):685-96. doi: 10.1002/path.4155

59. Wang YY, Jiang H, Pan J, Huang XR, Wang YC, Huang HF, et al. Macrophage-to-Myofibroblast Transition Contributes to Interstitial Fibrosis in Chronic Renal Allograft Injury. J Am Soc Nephrol (2017) 28(7):2053-67. doi: 10.1681/ASN.2016050573

60. Meng XM, Wang S, Huang XR, Yang C, Xiao J, Zhang Y, et al. Inflammatory Macrophages can Transdifferentiate Into Myofibroblasts During Renal Fibrosis. Cell Death Dis (2016) 7(12):e2495. doi: 10.1038/ cddis.2016.402

61. Kramann R, Machado F, Wu H, Kusaba T, Hoeft K, Schneider RK, et al. Parabiosis and Single-Cell RNA Sequencing Reveal a Limited Contribution of Monocytes to Myofibroblasts in Kidney Fibrosis. JCI Insight (2018) 3(9): e99561. doi: 10.1172/jci.insight.99561

62. Tan TK, Zheng G, Hsu TT, Lee SR, Zhang J, Zhao Y, et al. Matrix Metalloproteinase- 9 of Tubular and Macrophage Origin Contributes to the Pathogenesis of Renal Fibrosis Via Macrophage Recruitment Through Osteopontin Cleavage. Lab Invest (2013) 93(4):434-49. doi: 10.1038/ labinvest.2013.3

63. Wang X, Zhou Y, Tan R, Xiong M, He W, Fang L, et al. Mice Lacking the Matrix Metalloproteinase-9 Gene Reduce Renal Interstitial Fibrosis in Obstructive Nephropathy. Am J Physiol Renal Physiol (2010) 299(5):F973982. doi: 10.1152/ajprenal.00216.2010

64. Ren J, Zhang J, Rudemiller NP, Griffiths R, Wen Y, Lu X, et al. Twistl in Infiltrating Macrophages Attenuates Kidney Fibrosis Via Matrix Metallopeptidase 13-Mediated Matrix Degradation. J Am Soc Nephrol (2019) 30(9):1674-85. doi: 10.1681/ASN.2018121253 
65. Carlin LM, Stamatiades EG, Auffray C, Hanna RN, Glover L, Vizcay-Barrena G, et al. Nr4a1-Dependent Ly6C(low) Monocytes Monitor Endothelial Cells and Orchestrate Their Disposal. Cell (2013) 153(2):362-75. doi: 10.1016/ j.cell.2013.03.010

66. Westhorpe CLV, Norman MU, Hall P, Snelgrove SL, Finsterbusch M, Li A, et al. Effector CD4(+) T Cells Recognize Intravascular Antigen Presented by Patrolling Monocytes. Nat Commun (2018) 9(1):747. doi: 10.1038/s41467018-03181-4

67. Iglesias-Escudero M, Sansegundo-Arribas D, Riquelme P, Merino-Fernandez D, Guiral-Foz S, Perez C, et al. Myeloid-Derived Suppressor Cells in Kidney Transplant Recipients and the Effect of Maintenance Immunotherapy. Front Immunol (2020) 11:643. doi: 10.3389/fimmu.2020.00643

68. Pengam S, Durand J, Usal C, Gauttier V, Dilek N, Martinet B, et al. SIRPalpha/ CD47 Axis Controls the Maintenance of Transplant Tolerance Sustained by Myeloid-Derived Suppressor Cells. Am J Transplant (2019) 19(12):3263-75. doi: 10.1111/ajt.15497

69. Du XX, Guo YL, Zhao YP, Yang M, Chang S, Liu B, et al. Accumulation of High Levels of Monocytic Myeloid-Derived Suppressor Cells Enhances Graft Survival in Almost-Tolerant Kidney Transplant Recipients. Transplant Proc (2018) 50(10):3314-20. doi: 10.1016/j.transproceed.2018.04.043

70. Venet F, Monneret G. Advances in the Understanding and Treatment of Sepsis-Induced Immunosuppression. Nat Rev Nephrol (2018) 14(2):121-37. doi: 10.1038/nrneph.2017.165

71. Islam J, Lee HJ, Yang SH, Kim DK, Joo KW, Kim YS, et al. Expansion of MyeloidDerived Suppressor Cells Correlates With Renal Progression in Type 2 Diabetic Nephropathy. Immune Netw (2020) 20(2):e18. doi: 10.4110/in.2020.20.e18

72. Kapellos TS, Bonaguro L, Gemund I, Reusch N, Saglam A, Hinkley ER, et al. Human Monocyte Subsets and Phenotypes in Major Chronic Inflammatory Diseases. Front Immunol (2019) 10:2035. doi: 10.3389/fimmu.2019.02035

73. Heine GH, Ulrich C, Seibert E, Seiler S, Marell J, Reichart B, et al. CD14(++) CD16+ Monocytes But Not Total Monocyte Numbers Predict Cardiovascular Events in Dialysis Patients. Kidney Int (2008) 73(5):622-9. doi: 10.1038/ sj.ki.5002744

74. Rogacev KS, Seiler S, Zawada AM, Reichart B, Herath E, Roth D, et al. CD14+ +CD16+ Monocytes and Cardiovascular Outcome in Patients With Chronic Kidney Disease. Eur Heart J (2011) 32(1):84-92. doi: 10.1093/eurheartj/ehq371

75. Ramirez R, Carracedo J, Merino A, Soriano S, Ojeda R, Alvarez-Lara MA, et al. CD14+CD16+ Monocytes From Chronic Kidney Disease Patients Exhibit Increased Adhesion Ability to Endothelial Cells. Contrib Nephrol (2011) 171:57-61. doi: 10.1159/000327134

76. Lee JW, Cho E, Kim MG, Jo SK, Cho WY, Kim HK. Proinflammatory CD14 (+)CD16(+) Monocytes are Associated With Vascular Stiffness in Predialysis Patients With Chronic Kidney Disease. Kidney Res Clin Pract (2013) 32 (4):147-52. doi: 10.1016/j.krcp.2013.08.001

77. Geissmann F, Jung S, Littman DR. Blood Monocytes Consist of Two Principal Subsets With Distinct Migratory Properties. Immunity (2003) 19(1):71-82. doi: 10.1016/s1074-7613(03)00174-2

78. Stewart BJ, Ferdinand JR, Young MD, Mitchell TJ, Loudon KW, Riding AM, et al. Spatiotemporal Immune Zonation of the Human Kidney. Science (2019) 365(6460):1461-6. doi: 10.1126/science.aat5031

79. Kirita Y, Wu H, Uchimura K, Wilson PC, Humphreys BD. Cell Profiling of Mouse Acute Kidney Injury Reveals Conserved Cellular Responses to Injury. Proc Natl Acad Sci USA (2020) 117(27):15874-83. doi: 10.1073/pnas.2005477117
80. Zimmerman KA, Bentley MR, Lever JM, Li Z, Crossman DK, Song CJ, et al. Single-Cell RNA Sequencing Identifies Candidate Renal Resident Macrophage Gene Expression Signatures Across Species. J Am Soc Nephrol (2019) 30 (5):767-81. doi: 10.1681/ASN.2018090931

81. Belliere J, Casemayou A, Ducasse L, Zakaroff-Girard A, Martins F, Iacovoni JS, et al. Specific Macrophage Subtypes Influence the Progression of Rhabdomyolysis-Induced Kidney Injury. J Am Soc Nephrol (2015) 26 (6):1363-77. doi: 10.1681/ASN.2014040320

82. Dangi A, Natesh NR, Husain I, Ji Z, Barisoni L, Kwun J, et al. Single Cell Transcriptomics of Mouse Kidney Transplants Reveals a Myeloid Cell Pathway for Transplant Rejection. JCI Insight (2020) 5(20):e141321. doi: 10.1172/jci.insight.141321

83. Wang Y, Zhao Y, Zhao Z, Li D, Nie H, Sun Y, et al. Single-Cell RNA-Seq Analysis Identified Kidney Progenitor Cells From Human Urine. Protein Cell (2021) 12(4):305-12. doi: 10.1007/s13238-020-00816-5

84. de Zeeuw D, Bekker P, Henkel E, Hasslacher C, Gouni-Berthold I, Mehling H, et al. The Effect of CCR2 Inhibitor CCX140-B on Residual Albuminuria in Patients With Type 2 Diabetes and Nephropathy: A Randomised Trial. Lancet Diabetes Endocrinol (2015) 3(9):687-96. doi: 10.1016/S2213-8587(15)00261-2

85. Berthier CC, Zhang H, Schin M, Henger A, Nelson RG, Yee B, et al. Enhanced Expression of Janus Kinase-Signal Transducer and Activator of Transcription Pathway Members in Human Diabetic Nephropathy. Diabetes (2008) 58 (2):469-77. doi: 10.2337/db08-1328

86. Tuttle KR, Brosius FC3rd, Adler SG, Kretzler M, Mehta RL, Tumlin JA, et al. JAK1/JAK2 Inhibition by Baricitinib in Diabetic Kidney Disease: Results From a Phase 2 Randomized Controlled Clinical Trial. Nephrol Dial Transplant (2018) 33(11):1950-9. doi: 10.1093/ndt/gfx377

87. Tang TT, Lv LL, Wang B, Cao JY, Feng Y, Li ZL, et al. Employing Macrophage-Derived Microvesicle for Kidney-Targeted Delivery of Dexamethasone: An Efficient Therapeutic Strategy Against Renal Inflammation and Fibrosis. Theranostics (2019) 9(16):4740-55. doi: 10.7150/ thno. 33520

88. Potter SS. Single-Cell RNA Sequencing for the Study of Development, Physiology and Disease. Nat Rev Nephrol (2018) 14(8):479-92. doi: 10.1038/s41581-018-0021-7

89. Clatworthy MR. How to Find a Resident Kidney Macrophage: The Single-Cell Sequencing Solution. J Am Soc Nephrol (2019) 30(5):715-6. doi: 10.1681/ ASN.2019030245

90. Fu J, Akat KM, Sun Z, Zhang W, Schlondorff D, Liu Z, et al. Single-Cell RNA Profiling of Glomerular Cells Shows Dynamic Changes in Experimental Diabetic Kidney Disease. J Am Soc Nephrol (2019) 30(4):533-45. doi: 10.1681/ASN.2018090896

Conflict of Interest: The authors declare that the research was conducted in the absence of any commercial or financial relationships that could be construed as a potential conflict of interest.

Copyright (C) 2021 Wen, Yan, Wang and Liu. This is an open-access article distributed under the terms of the Creative Commons Attribution License (CC BY). The use, distribution or reproduction in other forums is permitted, provided the original author(s) and the copyright owner(s) are credited and that the original publication in this journal is cited, in accordance with accepted academic practice. No use, distribution or reproduction is permitted which does not comply with these terms. 